\title{
Videothoracoscopic management of a perforated central vein and pleura after ultrasound-guided internal jugular vein cannulation
} -a case report-

\author{
Jeong-Eun Kim, Joon-Pyo Jeon, Yongsuk Kim, Su Ah Jeong, and Young-Eun Moon \\ Department of Anesthesiology and Pain Medicine, Seoul St. Mary's Hospital, The Catholic University of Korea College of Medicine, \\ Seoul, Korea
}

A 23-year-old male underwent a left internal jugular vein catheterization during extended surgery for treatment of multiple fractures due to a traffic accident. Although the catheterization was performed under ultrasound (US) guidance, iatrogenic perforation of the central vein and pleura occurred. The catheter was removed, and the perforated site was addressed under thoracoscopy rather than an open thoracotomy. This case suggests that using US does not completely guarantee a complication-free outcome, and that catheter placement should be carefully confirmed. In addition, this case suggests that thoracoscopy may be an ideal method of resolving a perforation of the central vein and pleura. (Korean J Anesthesiol 2014; 66: 306-309)

Key Words: Central venous catheterization, Ultrasound, Video-assisted thoracoscopic surgery.

Insertion of a central venous catheter (CVC) is often performed in the operating room, but occasionally complications can be fatal $[1,2]$. Therefore, safe insertion with confirmation of correct placement of the catheter is vital. Ultrasound (US)-guided insertion of catheters has been used widely, and its safety and efficacy have been demonstrated in several studies. However, this technique is not free from complications. We report a case of a perforated central vein and pleura in a patient undergoing US-guided internal jugular vein (IJV) catheterization. Fortunately, the catheter, which acted as a plug after the perforation, was carefully removed under thoracoscopic guidance, instead of an open thoracotomy, and the perforations were repaired. Reports of thoracoscopic repair are rare, but we believe that this method was very useful. We report this case to show that complications can occur during US-guided IJV catheterization, as well as to discuss videothoracoscopic management as an ideal solution.

\section{Case Report}

A 23-year-old male (height: $184 \mathrm{~cm}$, weight: $64 \mathrm{~kg}$ ) was referred for general anesthesia and surgical repair of multiple

Received: March 19, 2013. Revised: April 19, 2013. Accepted: April 19, 2013.

Corresponding author: Young-Eun Moon, M.D., Ph.D., Department of Anesthesiology and Pain Medicine, Seoul St. Mary's Hospital, The Catholic University of Korea College of Medicine, 505, Banpo-dong, Seocho-gu, Seoul 137-701, Korea. Tel: 82-2-2258-6163, Fax: 82-2-537-1951, E-mail: 0910momo@naver.com

(c) This is an open-access article distributed under the terms of the Creative Commons Attribution Non-Commercial License (http:// creativecommons.org/licenses/by-nc/3.0/), which permits unrestricted non-commercial use, distribution, and reproduction in any medium, provided the original work is properly cited. 
fractures, including closed reduction of the nasal bone and open reduction and internal fixation of the right clavicle, right olecranon, and right femur shaft 1 week after a traffic accident. In addition, the patient sustained an extradural hematoma of the right fronto-temporal area, a flexion teardrop fracture of the $\mathrm{C} 7$ vertebra, a fracture of the second right rib, a right chest contusion, and a right pneumothorax. He had been receiving conservative management in the intensive care unit for 1 week. With the exception of increased aspartate aminotransferase/alanine aminotransferase of $43 / 50$, there were no notable abnormalities in the preoperative laboratory tests or electrocardiogram (ECG). As part of the evaluation for general anesthesia, the neurosurgeon recommended a neck brace with conservative care for the cervical spine and no additional treatment for the brain. The thoracic surgeon recommended continuing chest tube drainage of the right chest until the postoperative period.

The patient received total intravenous anesthesia (TIVA) rather than inhalation anesthesia, as he had a history of severe motion sickness. The patient was admitted to the operating room without preoperative medication. Anesthesia was induced after placement of the ECG, pulse oximeter, blood pressure monitor, and bispectral index (BIS) monitor. Propofol $(2 \mathrm{mg} / \mathrm{kg}$ ) and rocuronium $(0.8 \mathrm{mg} / \mathrm{kg})$ were administered, and endotracheal intubation was performed. Anesthesia was maintained with propofol and remifentanil in a mixture of air and $\mathrm{O}_{2}\left(\mathrm{FiO}_{2}=\right.$ 0.5 ). When the patient was admitted to the operating room, he had no intravenous lines, with the exception of a peripheral line in the left foot. As the operation was expected to be long, with potential for severe hemorrhage, we decided to place a central line. Because the right clavicle was one of the surgical sites, we decided to insert the CVC in the left IJV. The patient was placed in the Trendelenburg position to maintain the neck in a neutral position for increased spinal stability Ultrasound (S-Nerve, Sonosite, Inc., Bothell, WA, USA) was used to determine the location and anatomy of the left IJV, which was observed immediately above the left carotid artery. No thrombosis or other abnormality was noted. A second-year resident attempted the IJV puncture using an 18-G introducer needle (included in the Twolumen Central Venous Catheterization Set, Arrow International, Inc., Reading, PA, USA) with an out-of-plane (OOP) approach, taking care not to puncture the carotid artery immediately below. No blood return was observed on aspiration after the first puncture, but there was return of dark blood on aspiration after the second puncture. The US probe was removed, and a J-tipped wire guide was inserted via the introducer needle and advanced without resistance. The needle was removed and tissues were gently dilated without resistance with a dilator and a 2-lumen catheter (7 Fr). Blood return was confirmed on aspiration with a syringe, and the catheter was affixed at $18 \mathrm{~cm}$. The plastic surgeon began the nasal reduction immediately following insertion of the CVC. Forty minutes after induction of general anesthesia, the plastic surgery was completed, and the orthopedic surgeons took charge. The surgical site was re-draped, and the dorsal IV line for infusion of propofol and remifentanil was changed to the 18-G catheter of the CVC for further management. No notable hemodynamic instability or abnormal BIS signs had been observed until this time. However, the BIS began to increase gradually, $\sim 15 \mathrm{~min}$ into the first orthopedic surgery and reached 70. Midazolam ( $2 \mathrm{mg}$ ) was administered via the CVC to adjust the depth of anesthesia, followed by another $3 \mathrm{mg} 10 \mathrm{~min}$ later; however, no changes were observed. A central venous pressure (CVP) transducer was connected to monitor the CVP, and an abnormal waveform was observed. Because a CVC malfunction was suspected, the TIVA was discontinued, the anesthesia was switched to sevoflurane, and the BIS dropped to 40. When blood aspiration was attempted with a syringe to recheck placement of the CVC, a pinkish liquid returned instead of blood. Believing that this pinkish liquid was a mixture of propofol and blood, we obtained an emergency portable chest X-ray (CXR) to determine the exact position of the catheter, which revealed that the catheter was not in the left IJV but had perforated through the left pleura (Fig. 1). We determined that approximately $600 \mathrm{ml}$ of fluid had entered the thorax through the CVC and decided to consult a thoracic surgeon rather than directly remove the CVC. The thoracic surgeon recommended that an immediate thoracotomy was not required because the patient was hemodynamically stable and the CXR did not reveal a tension pneumothorax or hemothorax. However, considering possibilities such as damage to the adjacent blood vessels, they used thoracoscopy to confirm the position of the CVC. Furthermore, because we

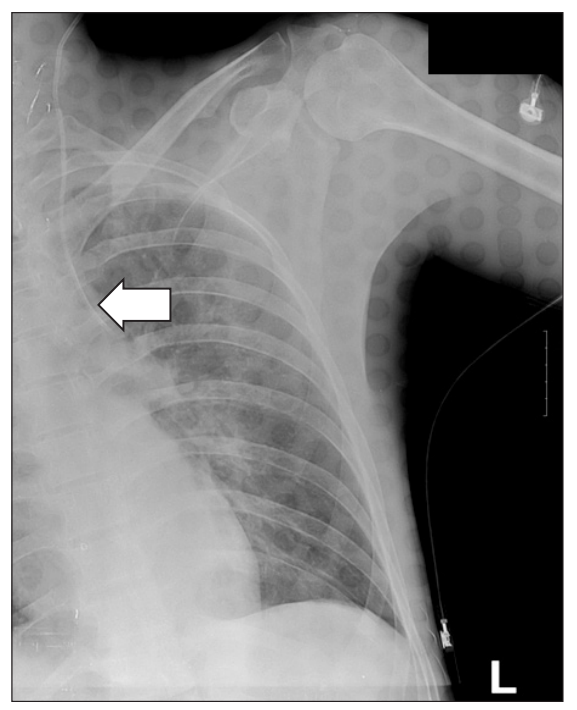

Fig. 1. The chest X-ray shows the central venous catheter (arrow) perforating through the central vein and the left pleura. 
suspected that the perforating catheter was acting as a plug, we planned to remove it under thoracoscopic guidance to reduce the risk of additional hemorrhage. The single-lumen endotracheal tube was switched to a left-sided double lumen tube (37 Fr) for thoracoscopy. Because the patient had multiple fractures on the right side, we attempted right tilting in the supine position, instead of a right decubitus position. After confirming that the patient had adapted well to one-lung ventilation, the surgeons began the thoracoscopy.

Under thoracoscopic guidance, we observed that the catheter had punctured the left upper pleura, and that there was a hematoma near the perforated catheter with no active bleeding. Furthermore, we observed an additional needle mark in the pleura near the catheter, which was thought to be from the first attempt. The hematoma near the catheter was removed with an endoscopic grasper, and gauze was endoscopically attached to compress the pleural orifice made by the catheter puncture. While the perforated site was compressed, the catheter was removed with external traction. Endoscopic compression was maintained at the perforation site for $10 \mathrm{~min}$ after removal of the catheter, and external compression of the cervical puncture site was applied concurrently. The perforation site was observed for $10 \mathrm{~min}$ after removing the gauze. When we confirmed that there was no active bleeding, the left chest tube was inserted, and the thoracoscopy was completed.

The tube was removed the following day, after we ensured that there was no further drainage into the left chest tube. Four days later, the patient underwent the orthopedic surgery that had been postponed from the previous day. He remained stable and was transferred to the general ward within a few days. The patient was ultimately discharged without further complications, with the exception of a stress ulcer.

\section{Discussion}

IJV catheterization is frequently used in the operating room or intensive care unit, but it is not free from complications. Commonly reported complications include carotid artery puncture, hemothorax, and pneumothorax. US-guided catheterization has been reported to effectively decrease complications compared to blind catheterization or the conventional landmark technique $[3,4]$. However, complications have been reported even when the procedure is guided by US $[5,6]$, and they can occur particularly during the learning curve of the technique.

The right IJV is usually preferred to the left IJV as the site for inserting a catheter because of the more direct route to the superior vena cava and the absence of the thoracic duct. However, we were unable to use the right IJV in this case, because it interfered with the surgical site. Furthermore, we attempted IJV catheterization using the OOP approach. The OOP approach is the most commonly used method for IJV catheterization and is particularly preferred by novice practitioners because it is much easier to perform than the in-plane (IP) technique and takes much less time. The IP technique, in which the needle can easily move out of plane and vanish from the screen, tends to seem more cumbersome and time consuming. Actually many studies evaluating real-time US-guided vascular access have used the OOP approach [7-9]. However, in an evaluation of US-guided IJV catheterization on a human torso mannequin using an OOP approach conducted with emergency medicine residents who were experienced in US-guided catheterization, the incidence of accidental needle penetration of the posterior vessel wall was $64 \%$, and the incidence of a final needle location deeper than the venous lumen and past the posterior wall was $24 \%$ [10]. Considering that the resident in our case had five previous experiences with US-guided catheterization, even experienced practitioners must be aware that the OOP approach carries a higher risk of penetration of the posterior wall than the IP approach. Furthermore, the IJV is highly superficial, and the vertical distance between the IJV and the skin may be $<2 \mathrm{~cm}$. Hence, if no flash of blood is observed after the needle has been inserted far deeper than expected, the needle should be removed and insertion should be attempted again. This is possible when the real field, as well as the sono field, is constantly monitored. However, novices may tend to focus too narrowly on the US view alone. Therefore, care should be taken lest they should miss the real field.

Methods of monitoring the correct placement of a CVC include fluoroscopy, confirmation by CXR, blood aspiration through the needle, monitoring changes on the ECG, confirmation of active venous return by lowering the drainage line to gravity, and evaluation of the CVP waveform. Generally, practitioners might tend to be less careful in the confirmation of correct placement when using US guidance compared to conventional blind techniques, as US may promote over-confidence. However, it should not be forgotten that the US view is twodimensional and a single image of a needle tip can correspond to a variety of locations (superior vena cava, innominate vein, or an extravascular location). In the current case, our resident confirmed the placement of the CVC only by initial aspiration of blood through the syringe, which we now believe was probably either from bleeding due to the posterior wall puncture or a hematoma by the first needle puncture. Indeed, it was not until the TIVA began to fail that we suspected a CVC malfunction. It is regrettable that we did not confirm placement by active regurgitation or evaluation of the CVP waveform, and we emphasize that proactive and unequivocal confirmation of placement is necessary even after US-guided insertion of a CVC.

Treatments and outcomes of pleural and vessel perforations by a CVC vary. If only a needle puncture has occurred, the problem is usually not critical. However, if large-bore catheter 
penetration and fluid injection are involved, complications such as hemothorax or hydrothorax may occur, and aggressive monitoring and consultation with a thoracic surgeon is required. If vital signs are unstable, an open thoracotomy should be performed immediately to correct the problem. However, in cases such as the one presented here, if vital signs are stable and no coagulopathy is present, a less-invasive thoracoscopy could be considered to assess the situation and correct the problem. Few reports of the benefits of thoracoscopic management of central venous injury are available $[11,12]$. However, as Kuzniec et al. [12] reported, there is no possibility of direct compression except by invasive thoracotomy, when there is an iatrogenic central vein perforation to the pleural space. If the catheter acts a plug for the perforated site for a relatively long period, withdrawal of a large-bore catheter without direct compression can lead to depacking of the injury and possibly to uncontrolled bleeding. In that situation, videothoracoscopy has value for both diagno- sis and treatment. Direct compression of the site at which the catheter perforated the vein and the pleura can be performed safely under videothoracoscopy. Similarly, in our case, thoracoscopy revealed that the catheter was acting as a plug. It should be noted that blind external removal of a malpositioned catheter, if it is acting as a plug, can lead to further complications such as hemothorax. Thus, although more time is required, confirmation through thoracoscopy is recommended.

In conclusion, practitioners should note the higher risk of posterior wall puncture with an OOP approach than an IP approach during US-guided IJV catheterization. Even US-guided CVC placement requires subsequent confirmation of correct catheter placement by proactive methods. Furthermore, examination and correction using videothoracoscopy can be an appropriate intervention more than open thoracotomy or videoscopic-assisted thoracotomy in cases of a catheter perforation

\section{References}

1. Dawood MM, Trebbin WM. Complications associated with central venous cannulation. Hosp Pract (Off Ed) 1991; 26: 211-4, 218-9.

2. McGee DC, Gould MK. Preventing complications of central venous catheterization. N Engl J Med 2003; 348: 1123-33.

3. Akoglu H, Piskinpasa S, Yenigun EC, Ozturk R, Dede F, Odabas AR. Real-time ultrasound guided placement of temporary internal jugular vein catheters: assessment of technical success and complication rates in nephrology practice. Nephrology (Carlton) 2012; 17: 603-6.

4. Karakitsos D, Labropoulos N, De Groot E, Patrianakos AP, Kouraklis G, Poularas J, et al. Real-time ultrasound-guided catheterisation of the internal jugular vein: a prospective comparison with the landmark technique in critical care patients. Crit Care 2006; 10: R162.

5. Jadhav AP, Stahlheber C, Hofmann H. Traumatic chyle leak: a rare complication of left internal jugular venous cannulation. Am J Med Sci 2011; 341: 238-9.

6. Parsons AJ, Alfa J. Carotid dissection: a complication of internal jugular vein cannulation with the use of ultrasound. Anesth Analg 2009; 109: 135-6.

7. Hrics P, Wilber S, Blanda MP, Gallo U. Ultrasound-assisted internal jugular vein catheterization in the ED. Am J Emerg Med 1998; 16: 4013.

8. Lamperti M, Subert M, Cortellazzi P, Vailati D, Borrelli P, Montomoli C, et al. Is a neutral head position safer than 45-degree neck rotation during ultrasound-guided internal jugular vein cannulation? Results of a randomized controlled clinical trial. Anesth Analg 2012; 114 : $777-$ 84.

9. Miller AH, Roth BA, Mills TJ, Woody JR, Longmoor CE, Foster B. Ultrasound guidance versus the landmark technique for the placement of central venous catheters in the emergency department. Acad Emerg Med 2002; 9: 800-5.

10. Blaivas M, Adhikari S. An unseen danger: frequency of posterior vessel wall penetration by needles during attempts to place internal jugular vein central catheters using ultrasound guidance. Crit Care Med 2009; 37: 2345-9.

11. Cheli M, Alberti D, Adriana T, Zaranko E, Colusso M, Arnoldi R, et al. Successful bleeding control by a combined conventional surgical approach and video-assisted surgery: a case report. Ann Thorac Cardiovasc Surg 2009; 15: 253-6.

12. Kuzniec S, Natal SR, Werebe Ede C, Wolosker N. Videothoracoscopic-guided management of a central vein perforation during hemodialysis catheter placement. J Vasc Surg 2010; 52: 1354-6. 